\title{
Successive Target Cancelation in Pulse Compression Radars
}

\author{
Onur Haliloğlu \\ TÜBITTAK-UZAY, \\ METU Campus, İnönü Bulvarı, 06531, \\ Balgat, Ankara, TÜRKIYE \\ phone: + (90) 312-2101310/1286, \\ email: onur.haliloglu@uzay.tubitak.gov.tr
}

\author{
A. Özgür Yilmaz \\ Dept. of Electrical-Electronics Eng. \\ Middle East Technical University, 06531, \\ Ankara, TÜRKIYE \\ phone: + (90) 312-2102337, \\ email: aoyilmaz@metu.edu.tr
}

\begin{abstract}
Many radars suffer from masking of weaker targets by stronger ones due to range sidelobes of pulse compression codes. We propose a method to prevent this by successively detecting targets and canceling their effects. Performance of the proposed method will be investigated in various scenarios with regard to existence of noise, targets, and the Doppler effect.
\end{abstract}

\section{INTRODUCTION}

Pulse compression is a widely used technique to avoid the limited peak power problem in radar systems. Pulse compression schemes are identical to spread spectrum systems in that detection is performed by a filter matched to the code of the pulse compression. However, range sidelobes are observed at the matched filter output due to non-ideal correlation properties. The sidelobes of a large target might be quite strong and mask smaller targets unless this issue is specifically taken into account.

Constant false alarm rate based algorithms have been used to prevent the above mentioned problem, though with little success [1]. Recently a couple of algorithms were proposed to remedy this problem with better success. Two of the most popular among these algorithms are the least squares (LS) estimation and the minimum mean square error (MMSE) estimation. In [2], [3], LS based methods are proposed whereas MMSE algorithm is run recursively in [4].

The LS algorithm [2], [3] estimates the received return samples by minimizing the squared errors between the observed received return samples and the estimated ones. As a result, the LS method is optimum in the squared error sense. LS estimation is performed by multiplying the received signal in the vector form by matrices obtained from code auto-correlation. Inversion of a $n \times n$ matrix is necessary in this method where $n$ is the length of the received signal. Although the matrix inversion is done off-line, the multiplication operation has an order of $O\left(n^{2}\right)$ and thus it is prohibitively computationally intensive. Hence, the greater the number of the samples taken for the received return signal is, the more computationally complex the LS algorithm becomes.

While running the MMSE algorithm [4], a filter is generated by using the matched filter (MF) output first to obtain target power estimates. A signal covariance matrix is obtained for each range bin to be inspected from target power estimates.
Another filter is constructed based on the inverse of the covariance matrix and acts on the MF signal. Smaller targets are identified by checking the filtered MF signal. At each iteration of the algorithm, target powers are reestimated and filters are regenerated. The algorithm is computationally complex due to on-line matrix inversion at every iteration. Furthermore, the estimated covariance matrix is reportedly ill-conditioned and thus ad hoc methods are used to avoid numerical instability in running the algorithm [4].

In [4], the LS and MMSE methods are compared. According to [4], the LS method suffers from lack of considering the entire set of received radar returns if a small window is used, since it does not account for the samples right before and after the window. If the window size is kept large, possibly covering all the received signals, the MMSE method has no benefit in performance when compared to the LS method. Therefore, in order to compare with our method we select the LS method whose window covers all the received returns.

Another alternative is obtaining the target's phasor from the MF output and canceling part of the signal due to the target. This can be repeated for each detected target. Since this method successively detects a signal and cancels the signal from the overall MF output, we will refer to it as Successive Target Cancelation (STC). STC is based on basic operations such as addition and subtraction. It does not include matrix inversion or other complex operations and hence is simple and easy to handle. This algorithm known as the CLEAN algorithm [5] was applied in image processing [6], [7] and astronomy [8], [9] as a deconvolution method. A similar method is called Successive Interference Cancelation in communication literature [10] and is usually employed for multi-user detection. We will investigate the performance of this approach for radar applications in this report.

The outline of the paper is as follows. We describe the method by analyzing a two target scenario in Section 2 . The simulation results are presented in Section 3. The paper concludes with Section 4. 


\section{Successive Estimation And CANCElation}

In this section we will try to illustrate the basic idea of the proposed method. In radar applications, the received signal $y(k)$ is passed through the matched filter and then the CLEAN algorithm is applied to the matched filter output $m(k)$. The representation of discrete time forms of the received signal and the MF output are respectively given below:

$$
\begin{aligned}
& y(k)=h(k) * s(k)+w(k), \\
& m(k)=y(k) * s^{*}(-k),
\end{aligned}
$$

where $s(k)$ is the transmitted signal, $w(k)$ is the additive noise, and ' $*$ ' denotes the convolution operation. The basic CLEAN algorithm is presented in its entirety in Table 1.

TABLE I

STC ALGORITHM

1) Obtain the MF output $m(k)$, initialize all estimates $h(k)$ to zero.

2) Find the peak in $|m(k)|>\tau$. If no such point exists, halt.

3) If the peak exceeds $\tau$ and its location is $u$ so that $|m(u)|$ is the largest, estimate the target at $k=u$ by $h(u)=h(u)+\lambda \frac{m(u)}{r(0)},(1 \geq \lambda>0)$.

4) Cancel the effect of the most recently estimated target by $m(k)=m(k)-\lambda \frac{m(u)}{r(0)} r(k-u)$

5) Go to step 2 .

The threshold $\tau$ is important for detection of radar signals. Moreover, as parameter $\lambda$, it directly affects the computational complexity of the algorithm. In this study, we take the $\tau$ to be equal to the constant false alarm rate (FAR) threshold for the noise at the MF output for a chosen FAR.

In order to clarify the basic idea of the CLEAN algorithm, it is beneficial to investigate a simplified radar scenario. Therefore, the simplest scenario where only two targets exist both at zero Doppler frequency will be studied. The effect of noise will be taken into consideration whereas clutter is neglected in this section. For simplicity, the value of $\lambda$ is taken as 1 . We consider the case that the power difference between two targets is larger than the code sidelobes so that the weak target cannot be detected by simply checking the matched filter output.

Suppose we send a radar pulse with

$$
s(t)=\sum_{p=0}^{L-1} a_{p} g\left(t-p T_{c}\right),
$$

where $L$ is the number of chips in the code, $a_{p}$ 's denote the code sequence, $g(\cdot)$ is taken to be the rectangular pulse in $\left[0, T_{c}\right]$, and $T_{c}$ is the chip duration. Assume we have two targets whose signals arrive $k_{1}$ and $k_{2}$ (both are assumed to be integers) chip durations later after we send a pulse. Thus, we have the following discrete-time impulse response observed by the receiver

$$
h(k)=\alpha_{1} \delta\left(k-k_{1}\right)+\alpha_{2} \delta\left(k-k_{2}\right),
$$

where $\alpha_{1}$ and $\alpha_{2}$ are the phasors of targets 1 and 2, respectively. Rather than using the continuous time notation for the signals, we will use the discrete time baseband equivalent model exemplified in (2) throughout the paper. The phasors are circularly symmetric zero-mean complex Gaussian random variables with high enough signal-to-noise ratios (SNR) for detection. So, target powers are quite above the noise level.

The received signal is given by

$y(k)=h(k) * s(k)+w(k)=\alpha_{1} s\left(k-k_{1}\right)+\alpha_{2} s\left(k-k_{2}\right)+w(k)$,

where $*$ denotes the convolution operation and the front-end receiver filters and samplers are constructed in a way that $w(k)$ is an additive white Gaussian noise (AWGN) at time $k$. The AWGN is taken to be circularly symmetric complex Gaussian with mean 0 and variance $N_{0}$. The received signal is passed through a matched filter (MF). Neglecting the time shift for causality in MF, we obtain the MF output $m(k)$ by

$m(k)=y(k) * s^{*}(-k)=\alpha_{1} r\left(k-k_{1}\right)+\alpha_{2} r\left(k-k_{2}\right)+n(k)$,

where $n(k)=w(k) * s^{*}(-k)$ and the autocorrelation of $s(k)$ is $r(k)=s(k) * s^{*}(-k)$.

Recalling that $\left|\alpha_{1}\right|^{2}>\left|\alpha_{2}\right|^{2}$, the largest peak of $|m(k)|$ will be observed at $k=k_{1}$ with high probability since the noise power is very small compared to target powers. The MF output at $k=k_{1}$ is

$$
m\left(k_{1}\right)=\alpha_{1} r(0)+\alpha_{2} r\left(k_{1}-k_{2}\right)+n\left(k_{1}\right)
$$

and there is a priori information on neither the powers nor the time shifts of targets. Then, the best estimate we can have for $\alpha_{1}$ is

$$
\hat{\alpha}_{1}=\frac{m\left(k_{1}\right)}{r(0)}=\alpha_{1}+\alpha_{2} \frac{r\left(k_{1}-k_{2}\right)}{r(0)}+\frac{n\left(k_{1}\right)}{r(0)} .
$$

We thus obtain an estimate of $\alpha_{1}$ which is biased. The mean of the estimate has an additive term affected by $\alpha_{2}$, though its effect is through the sidelobes so that the code employed reduces this effect.

We will now subtract the signal that is due to target 1 using its phasor estimate. This can be done both at the MF output or the video signal. Although the results are identical in the ideal case due to linearity, issues such as quantization is important in deciding which one is to be preferred to practice. We will perform cancelation at MF output in this study. Denote this new signal by $m^{\prime}(k)$ such that

$$
\begin{aligned}
m^{\prime}(k)= & m(k)-\hat{\alpha}_{1} r\left(k-k_{1}\right) \\
= & \alpha_{2} r\left(k-k_{2}\right)-\alpha_{2} \frac{r\left(k_{1}-k_{2}\right)}{r(0)} r\left(k-k_{1}\right)+n(k) \\
& -\frac{n\left(k_{1}\right)}{r(0)} r\left(k-k_{1}\right) .
\end{aligned}
$$

We now like to detect target 2 based on this updated MF signal. As seen in (7), the largest peak of $\left|m^{\prime}(k)\right|$ will be at $k=k_{2}$ 
with high probability. At $k=k_{2}$,

$m^{\prime}\left(k_{2}\right)=\alpha_{2} r(0)-\alpha_{2} \frac{\left|r\left(k_{1}-k_{2}\right)\right|^{2}}{r(0)}+n\left(k_{2}\right)-\frac{r\left(k_{2}-k_{1}\right)}{r(0)} n\left(k_{1}\right)$

so that the phasor of target 2 can be estimated as

$$
\begin{aligned}
\hat{\alpha}_{2}= & \frac{m^{\prime}\left(k_{2}\right)}{r(0)} \\
= & \alpha_{2}-\alpha_{2} \frac{\left|r\left(k_{1}-k_{2}\right)\right|^{2}}{r^{2}(0)}+\frac{1}{r(0)} n\left(k_{2}\right) \\
& -\frac{r\left(k_{2}-k_{1}\right)}{r^{2}(0)} n\left(k_{1}\right) .
\end{aligned}
$$

There is no contribution from the first target in $\hat{\alpha}_{2}$ as shown in (9). The second term with $\alpha_{2}$ has a squared multiplicative term and is very small compared to the first term when the maximum sidelobe levels of pulse compression codes used in practice are considered. The same argument holds for the second noise term. So, the signal-to-noise ratio (SNR) for $\alpha_{2}$ approaches the SNR value $\frac{\left|\alpha_{2}\right|^{2} r(0)}{N_{0}}$. If there was only target 2 in the system, we would have the same SNR for the estimate of its phasor. This suggests that the masking problem due to sidelobes is alleviated to a large extent with the method explained in this section.

At this point we have a good phasor estimate for target 2 and can subtract its effect from the MF output. The reason of such an iteration is the existence of a term related to $\alpha_{2}$ in $\hat{\alpha}_{1}$. Though weaker compared to target 1 , target 2 still has sidelobes and it affected the phasor estimate of target 1 as seen in (6). This overshooting may be corrected by another iteration of the algorithm. When target 2 is canceled from the MF output currently held by the algorithm, the following is obtained:

$$
\begin{aligned}
m^{\prime \prime}(k)= & m^{\prime}(k)-\hat{\alpha}_{2} r\left(k-k_{2}\right) \\
= & -\frac{r\left(k_{1}-k_{2}\right)}{r(0)} \alpha_{2} r\left(k-k_{1}\right) \\
& +\frac{\left|r\left(k_{1}-k_{2}\right)\right|^{2}}{r^{2}(0)} \alpha_{2} r\left(k-k_{2}\right)+n(k) \\
& -\frac{n\left(k_{1}\right)}{r(0)} r\left(k-k_{1}\right)-\frac{n\left(k_{2}\right)}{r(0)} r\left(k-k_{2}\right) \\
& +\frac{r\left(k_{2}-k_{1}\right) n\left(k_{1}\right)}{r^{2}(0)} r\left(k-k_{2}\right) .
\end{aligned}
$$

Once again, $\left|m^{\prime \prime}(k)\right|$ has its peak at $k=k_{1}$ with high probability and we have the chance to estimate the overshoot term by $\frac{m^{\prime \prime}\left(k_{1}\right)}{r(0)}$. We will now add this overshoot term to the current estimate for $\alpha_{1}$ :

$$
\begin{aligned}
\hat{\alpha}_{1}^{\prime}= & \hat{\alpha}_{1}+\frac{m^{\prime \prime}\left(k_{1}\right)}{r(0)} \\
= & \alpha_{1}+\frac{r\left(k_{1}-k_{2}\right)\left|r\left(k_{1}-k_{2}\right)\right|^{2}}{r^{3}(0)} \alpha_{2} \\
& +\left[1+\frac{\left|r\left(k_{1}-k_{2}\right)\right|^{2}}{r^{2}(0)}\right] \cdot \frac{n\left(k_{1}\right)}{r(0)} \\
& -\frac{r\left(k_{1}-k_{2}\right)}{r(0)} \cdot \frac{n\left(k_{2}\right)}{r(0)} .
\end{aligned}
$$

When this new estimate is carefully examined and compared to (6), it can be seen that the effect of target 2 is now reduced by the $\frac{\left|r\left(k_{1}-k_{2}\right)\right|^{2}}{r^{2}(0)}$ term whereas the noise terms almost have the same variance.

The overshoot terms added at later iterations act as correction terms and enhance the estimation. The iterations can be repeated as many times as desired. However, the estimates will be very accurate after some time and the correction terms will be only due to noise. That's why we suggest only the correction terms which can pass a certain threshold be taken into consideration. This also gives way to a stopping criterion for the algorithm.

The STC method is based on simple operations such as addition, multiplication etc. whereas LS has to deal with matrix multiplication with a large matrix. Thus, the STC algorithm is less complex than the LS algorithm. Similarly, STC has smaller complexity compared to the MMSE method in [4] due to both the matrix inversions and the large number of bins for which the algorithm has to be run for MMSE even in the single target case.

If a target has non-zero Doppler then the received signal is distorted due to frequency shift. This distortion has to be taken into consideration. In the STC method, after the MF output is obtained from the received return signal, we assume for simplicity that fast Fourier transform (FFT) is applied to the MF output for the Doppler processing. The STC algorithm can be run for each FFT filter. By checking the maximum absolute signal in a filter, it can be determined whether at least a single target is present in that filter. If at least one target exists, then the STC algorithm is run for that filter. In the STC method, the distortion due to Doppler is taken into account by modifying the auto-correlation $c(k)$. In this case, the auto-correlation is taken as

$$
c(k)=\left(e^{j 2 \pi P R F \cdot T_{c} \cdot \frac{b-1}{K}(0: K-1)} \otimes s(k)\right) * s^{*}(-k),
$$

where $b$ corresponds to the filter number, PRF is the pulse repetition frequency, $K$ is the number of pulses in a burst, and $\otimes$ denotes the Kronecker product. This modification stems from the fact that the received signal due to a target with nonzero Doppler is shifted in frequency. For the $b^{\text {th }}$ FFT filter, it is hypothesized that the Doppler shift equals $(b-1) \frac{P R F \cdot T_{c}}{K}$.

In the LS method, FFT has to be applied directly to the received return signal since MF operation takes place within LS. In order to determine which FFT filter output to use, it is necessary to check all FFT filters since MF is not applied prior to FFT.The LS algorithm run for the $b^{t h}$ FFT filter takes the received signal as the Doppler shifted one and thus calculates the correlation matrix.

\section{SIMULATION RESULTS}

To demonstrate the performance of the STC algorithm in comparison to the LS method we examine mainly two cases. In both cases the transmitted waveform is the $P 4$ code [11] of length $N=30$, the parameter $\lambda$ is taken as 1 , and the noise is modeled as a white circularly symmetric complex Gaussian 
noise with power spectral density $N_{0}=10^{-4}$. The length of the environment response is 90 chips.

In all of the simulations, the fast Fourier transform (FFT) in its plain form, i.e., no windowing, is applied to the matched filter output to account for Doppler processing. The number of transmitted pulses is 8 , so the number of FFT filters is also 8. The pulse repetition frequency (PRF) is $8000 \mathrm{~Hz}$ and the threshold $\tau$ in STC is set for a false alarm rate (FAR) of 0.1 unless specifically defined.

\section{A. Multiple Targets Case}

In this case, we investigate the relation between the target density and the residual error power. Residual error power is defined as the power of the difference signal between the true radar impulse response and the estimated radar impulse response. We use the target density parameter to indicate the number of targets in a given environment. Through this scenario, we can investigate the performance at the zero Doppler filter where many impulses due to clutter, which can be considered as targets around zero velocity, are expected. Moreover, multiple targets at other filters can be inspected within this scenario as well. When the target density is zero, there is no target in the environment. When it is 1 , there exists a target signal in each range bin. The target signals are modeled as circularly symmetric zero-mean complex Gaussian random variables with variance 1 . The noise and target signals are formed 1000 times randomly for each target density value. For this case, first one of the eight FFT filters, which operates in between $-500(7500)-500 \mathrm{~Hz}$, is used. The residual error performance of the STC and LS algorithms are compared in Figures $1-4$.

In Figure 1, it can be seen that for zero Doppler the performance of the STC method leads LS method approximately up to target density of 0.35 . For the values of target density above 0.35 , LS method is obviously better than STC method. However, it is highly unlikely that more than half of the range bins at a given direction are filled with the target signals even for the zero-Doppler filter.

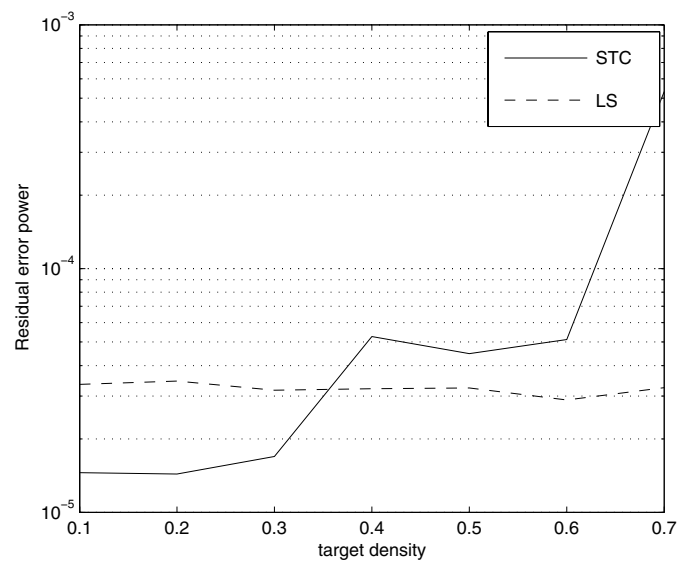

Fig. 1. Residual error performance of LS and STC methods with respect to target density at zero Doppler.
When there is Doppler on targets, STC performance comes even closer to LS performance. As seen in Figures 2 and 3, when the Doppler frequency increases, the performances of both methods deteriorate. However, the performance decrease in STC is less than the performance decrease in LS. It can be concluded that with respect to the residual error power performance, STC can be preferred against LS when the targets do not occupy more than half of the range bins.

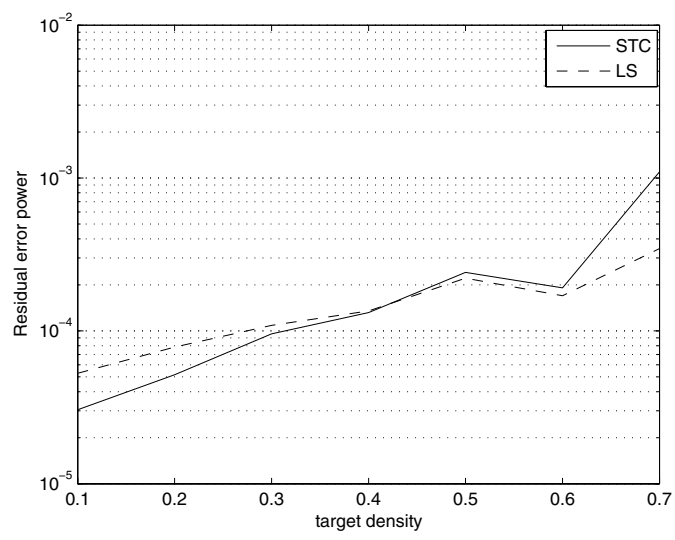

Fig. 2. Residual error performance of LS and STC methods with respect to target density when Doppler frequencies of targets are randomly distributed with the uniform density between -200 and $200 \mathrm{~Hz}$.

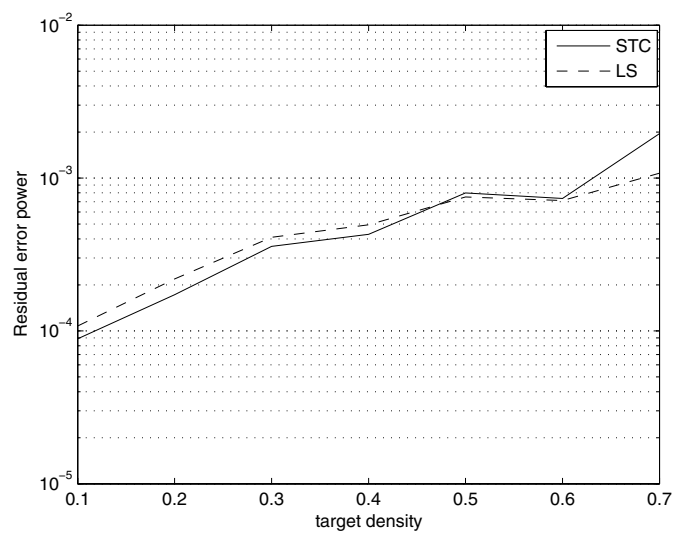

Fig. 3. Residual error performance of LS and STC methods with respect to the target density when Doppler frequencies of targets are randomly distributed with the uniform density between -500 and $500 \mathrm{~Hz}$.

In Figure 4, the third FFT filter is observed since the Doppler frequency of the targets is distributed between 1500 and $2500 \mathrm{~Hz}$. The residual error performances of Figures 3 and 4 are not very different. When the correlation function is corrected for the center frequency of the studied filter as explained in the last section, it can be concluded that the STC algorithm performs well even Doppler effect exists.

Actually, the residual error performance of any method can not be better than that of the LS method because residual error power is equivalent to the cost function of the LS method and LS method is optimum in the mean squared error sense 


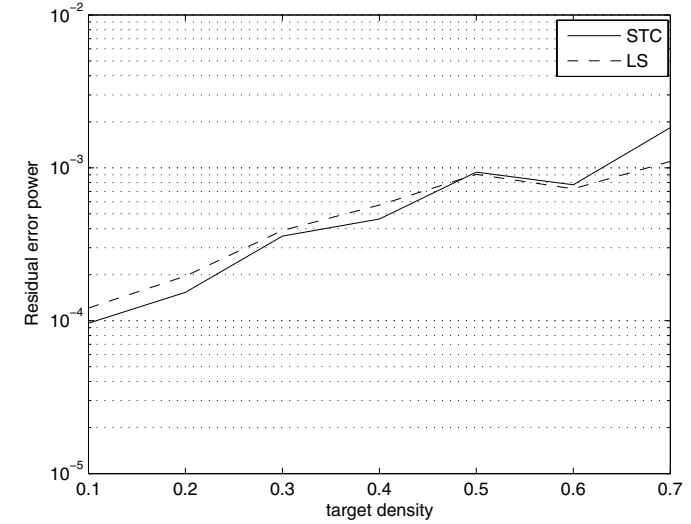

Fig. 4. Residual error performance of LS and STC methods with respect to the target density when Doppler frequencies of targets are randomly distributed with the uniform density between 1500 and $2500 \mathrm{~Hz}$.

for an unbiased estimation. However, the missing point is that our method STC obtains a biased estimate. Therefore, residual error performance of the STC method can be better than that of the LS method. Figure 5 demonstrates the biasedness of the STC algorithm. In this figure, STC algorithm is run 50, 250, and 10000 times for the same 9 targets in the environment and then averaged. Target signals with zero Doppler are deterministic and target locations are constant. The difference, namely the error, between the true radar impulse response and the mean of the estimated impulse response. The real and imaginary parts of the error are drawn. As seen in Figure 5 , as the number of Monte Carlo runs used in averaging increases the random errors tend to diminish in most range bins. However, at bins where there are targets present the expected value of the error does not change. Hence, we can conclude that STC is a biased estimation algorithm.
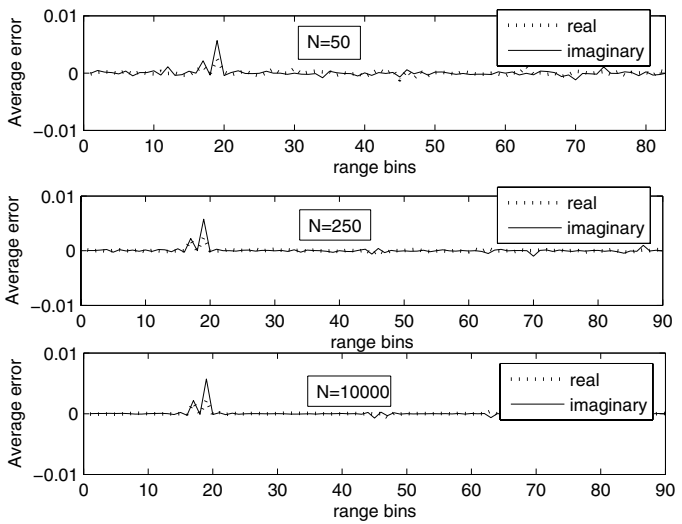

Fig. 5. Real and imaginary parts of the average error of the STC method for $N=50,250$, and 10000 .

\section{B. Two Targets Case}

In the second case, there is a large target and a small target in the environment. The small target is masked by the range sidelobes of the large target. We will check out the probability of detection $\left(P_{d}\right)$ performance versus signal to noise ratio (SNR) of the small target. In many radar applications, targets are in motion. Therefore, Doppler effect is taken into account in this case in order to be more realistic. The third FFT filter which operates in between $1500-2500 \mathrm{~Hz}$ is used.

STC aims to eliminate the adverse effects of sidelobes by estimating and canceling target signals. In order to observe whether STC can resolve the masking problem, we will compare the small-large target case (a) with the case that there is no masking (b), i.e., only the small target is present. If two cases have similar Pd performance, then STC will have fulfilled its promise.

In Figure 6, both target signals are deterministic, have fixed powers, and are 7 range bins apart from each other. In this figure, the power of the large target is $28 \mathrm{~dB}$ higher than the power of the small target. The Doppler frequencies of the large target and the small target are $2200 \mathrm{~Hz}$ and $1700 \mathrm{~Hz}$ respectively. The noise power $N_{0}$ is varied so that the SNR effect on Pd can be examined. 1000 samples are taken for each $N_{0}$ value. The performance of detection based only on MF output is also depicted for comparison and bears virtually no difference with STC. As seen in Figure 6, whether the large target is present in the environment or not, there is not much difference in Pd of the small target between LS and STC methods. Moreover, when STC method is applied, Pd of the small target does not change much according to the existence of the large target.

In Figure 7, the small target signal is formed randomly with a complex Gaussian distribution for each 1000 samples while the large target is deterministic. The SNR of the big target is $50 \mathrm{~dB}$ and $N_{0}$ of the noise is constant. However, noise and small target signal are randomly generated. In this figure, STC and LS methods give so close results that Pd-SNR curves of the methods can not easily be differentiated. Pd-SNR curve of the STC method in the existence of a large target is nearly the same as the one when the large target does not exist.

In conclusion, it is obviously observed from the two figures that STC reduces most of the adverse effects of the sidelobe of the large target. Indeed, STC almost achieves optimum sidelobe reduction in the squared error sense with a reasonable complexity.

The effect of the parameter $\lambda$ in the CLEAN algorithm is also investigated. However, the corresponding figures are not put into this paper due to the lack of space. Considering that $\lambda$ does not affect the residual error performance much,generally in the multiple target case, the residual error power of the STC method increases as $\lambda$ decreases. On the other hand, for the smaller target density values higher $\lambda$ leads to bigger residual errors. In the two target case, $P_{d}$ performances of small $\lambda$ values are worse. Moreover, according to other simulations based on the effects of $\lambda$ on the proposed method, it can be concluded that $\lambda$ increases the complexity and the false alarms. 

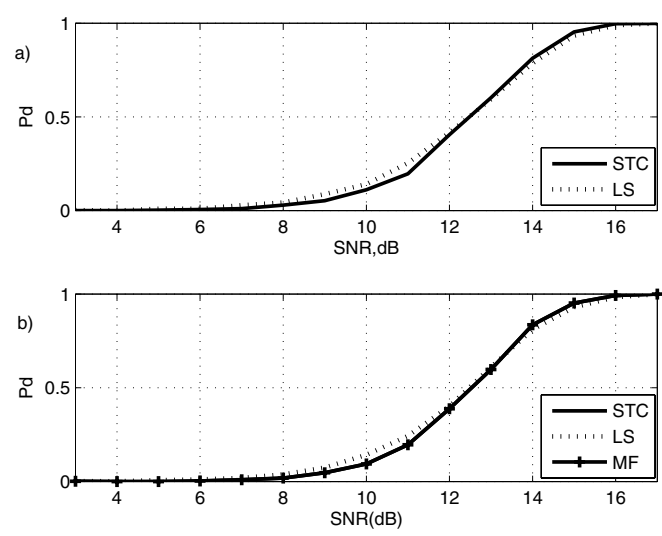

Fig. 6. Probability of detection versus signal to noise ratio is examined for a) A deterministic small target masked by a deterministic large target whose power is $28 \mathrm{~dB}$ higher than the small one. b) Only a deterministic small target
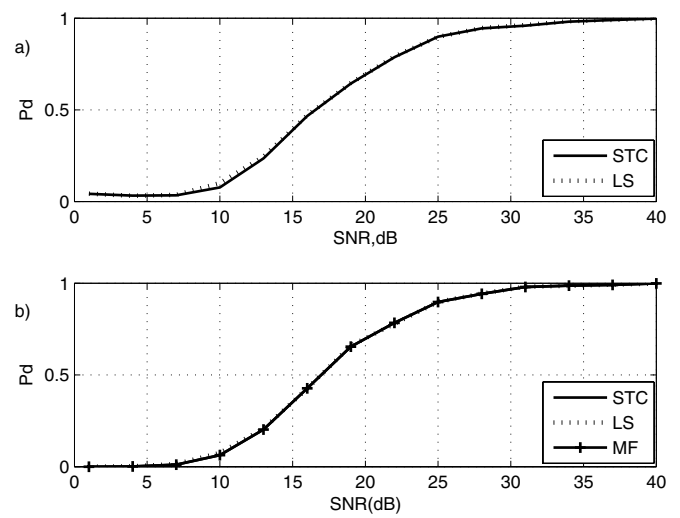

Fig. 7. Probability of detection versus signal to noise ratio is examined for a) A randomly formed small target and a deterministic large target. b) Only a random small target

\section{CONCLUSION}

Pulse compression is ubiquitously used in radar applications. The standard matched filtering not only solves the limited peak power problem but also maximizes the received SNR of the target in the existence of white noise. However, this technique has the problem of sidelobe masking of smaller targets in the vicinity of large targets due to non-ideal correlation properties. To overcome this problem, we propose a new method called Successive Target Cancelation (STC). Basically, the STC method detects target signals and cancels them out from the matched filter output successively.

According to the simulation results, STC performs well as long as the number of targets in the received signal is not excessively large. The Doppler effect can be easily taken into account in STC without much degradation in performance. In general, the successive target cancelation (STC) method almost carries out the optimum performance in the squared error sense. Moreover, it has lower complexity compared to other methods. Hence, it presents a viable solution to sidelobe problem in pulse compression radars.

\section{REFERENCES}

[1] M. I. Skolnik, Introduction to Radar Systems, McGraw-Hill, 1980.

[2] S. Moon-Ho Song, Woonkyurig M. Kim and Dongwook Park and YoungSik Kim, Estimation theoretic approach for radar pulse compression processing and its optimal codes, Electronics Letters, vol. 36,no. 3, pp. 250-252, Feb. 2000.

[3] T. K. Sarkar and R. D. Brown, An ultra-low sidelobe pulse compression technique for high performance radar systems, Proc. IEEE National Radar Conf., pages 111-114, May 1997.

[4] S. D. Blunt and K. Gerlach, Adaptive Pulse Compression, IEEE National Radar Conference, pp. 271-276, 2004

[5] U. J. Schwarz, Mathematical-statistical Description of the Iterative Beam Removing Technique (Method CLEAN), Astron. Astrophys., vol. 65, pp. 345-356, Feb. 1978.

[6] S. Horiuchi, S. Kameno, and M. Ohishi, Developing a Wavelet CLEAN Algorithm for Radio-Interferometer Imaging, Astronomical Data Analysis Software and Systems X ASP Conference Series, vol. 238, 2001.

[7] G. M. Skofronick-Jackson and A. J. Gasiewski, A nonlinear multispectral statistical CLEAN-based precipitation parameter-retrieval algorithm, Geoscience and Remote Sensing, IEEE Transactions on, vol. 38, no. 1, pp. 226-237, Jan. 2000.

[8] J. L. Starck, E. Pantin, and F. Murtagh, Deconvolution in astronomy: A review, Publications of the Astronomical Society of the Pacific, vol. 114, pp. 1051-1069, Oct. 2002.

[9] M. Peichl, M. Greiner, and H. Suess, DLR activities on aperture synthesis radiometry, Geoscience and Remote Sensing Symposium, 2000. Proceedings. IGARSS 2000. IEEE 2000 International, vol. 7, pp. 2981-2983, July. 2000 .

[10] S. Verdu, Multiuser Detection, Cambridge University, 1998.

[11] F. F. Kretschmer and K. R Gerlach, Low Sidelobe Pulse Compression Waveforms, IEEE Signals, Systems and Computers Conference, vol. 2, pp. 663-667, Nov. 1988 